\title{
BMJ Open High-dose vitamin D supplementation to prevent prostate cancer progression in localised cases with low-to-intermediate risk of progression on active surveillance (ProsD): protocol of a phase II randomised controlled trial
}

\author{
Visalini Nair-Shalliker (D) , ${ }^{1,2,3}$ David P Smith, ${ }^{1,3}$ Val Gebski, ${ }^{4}$ Manish I Patel,,${ }^{3,5}$ \\ Mark Frydenberg, ${ }^{6}$ John W Yaxley, ${ }^{7,8}$ Robert Gardiner, ${ }^{7}$ David Espinoza, ${ }^{4}$ \\ Michael G Kimlin, ${ }^{9}$ Michael Fenech, ${ }^{10}$ David Gillatt, ${ }^{2}$ Henry Woo, ${ }^{3,11}$ \\ Bruce K Armstrong, ${ }^{12}$ Krishan Rasiah, ${ }^{3,13}$ Nader Awad, ${ }^{14,15}$ James Symons, ${ }^{3,11}$ \\ Howard Gurney ${ }^{2}$
}

To cite: Nair-Shalliker V, Smith DP, Gebski V, et al. Highdose vitamin $D$ supplementation to prevent prostate cancer progression in localised cases with low-to-intermediate risk of progression on active surveillance (ProsD): protocol of a phase II randomised controlled trial. BMJ Open 2021;11:e044055. doi:10.1136/ bmjopen-2020-044055

- Prepublication history and supplemental material for this paper is available online. To view these files, please visit the journal online (http://dx.doi. org/10.1136/bmjopen-2020044055).

Received 24 August 2020 Revised 16 December 2020 Accepted 04 January 2021

Check for updates

(C) Author(s) (or their employer(s)) 2021. Re-use permitted under CC BY-NC. No commercial re-use. See rights and permissions. Published by BMJ.

For numbered affiliations see end of article.

Correspondence to Dr Visalini Nair-Shalliker; visalinin@nswcc.org.au

\section{ABSTRACT}

Introduction Active surveillance (AS) for patients with prostate cancer $(\mathrm{PC})$ with low risk of $\mathrm{PC}$ death is an alternative to radical treatment. A major drawback of AS is the uncertainty whether a patient truly has Iow risk PC based on biopsy alone. Multiparametric MRI scan together with biopsy, appears useful in separating patients who need curative therapy from those for whom AS may be safe. Two small clinical trials have shown short-term high-dose vitamin D supplementation may prevent PC progression. There is no substantial evidence for its long-term safety and efficacy, hence its use in the care of men with PC on AS needs assessment. This protocol describes the ProsD clinical trial which aims to determine if oral high-dose vitamin D supplementation taken monthly for 2 years can prevent PC progression in cases with low-to-intermediate risk of progression.

Method and analysis This is an Australian national multicentre, 2:1 double-blinded placebo-controlled phase II randomised controlled trial of monthly oral high-dose vitamin D supplementation (50 $000 \mathrm{IU}$ cholecalciferol), in men diagnosed with localised PC who have low-to-intermediate risk of disease progression and are being managed by AS. This trial will assess the feasibility, efficacy and safety of supplementing men with an initial oral loading dose of $500000 \mathrm{IU}$ cholecalciferol, followed by a monthly oral dose of $50000 \mathrm{IU}$ during the 24 months of AS. The primary trial outcome is the commencement of active therapy for clinical or non-clinical reason, within 2 years of AS.

Ethics and dissemination This trial is approved by Bellberry Ethics Committee (2016-06-459). All results will be reported in peer-reviewed journals. Trial registration number ACTRN12616001707459.

\section{Strengths and limitations of this study}

This double-blinded placebo controlled randomised controlled trial with stringent allocation concealment eliminates treatment and allocation bias.

- Adherence to the trial is likely high due to regular patient follow-ups and mode of delivery of intervention by mail makes this trial accessible to men in urban and rural areas.

- Blood collection at varying time points makes this a valuable resource but is costly.

- This is a phase 2 trial, hence results will not be conclusive for the role of vitamin $D$ but may inform a phase 3 trial, is a limitation.

- Follow-up is 2 years and therefore a benefit or lack of benefit of vitamin D may not be appreciated in this time, given the natural slow progression of prostate cancer, is a limitation.

\section{INTRODUCTION}

Prostate cancer (PC) affects many men worldwide, with 1276106 new cases and 358989 deaths estimated for $2018 .^{12}$ The proportion of low-risk tumours diagnosed has risen since the introduction of prostate-specific antigen (PSA) testing, leading in some cases to overdiagnosis, adverse effects and unnecessary treatment. ${ }^{3}$ Over diagnosis due to PSA-testing may be unavoidable, but overtreatment is not. Results from the prostate testing for cancer and treatment suggest minimal benefits in men with low-risk PC when managed with curative treatment, and instead recommended monitoring the course of PC with the intention of initiating curative treatment 
if and when the cancer progresses. Referred to as active surveillance (AS), this management option has evolved as an alternative to immediate active treatment for those diagnosed with low grade disease. ${ }^{4}$ Consequently, AS is now the preferred management strategy for most men with low-risk PC. It aims to avoid overtreatment of clinically indolent disease by safely delaying definitive treatment until evidence of progression is evident.

The following augmented risk classification for low, intermediate and high-risk groups is used as guide to classify disease status ${ }^{5-7}$ :

- Low risk: PSA $<10 \mathrm{ng} / \mathrm{mL}$, and Gleason $3+3$ or less, 2 or less positive biopsy cores and clinical stage T1-T2a.

- Intermediate: PSA 10 to $<20 \mathrm{ng} / \mathrm{mL}$ or Gleason 7 (Gleason $3+4$ or Gleason 4+3) or clinical stage T2b-c subdivided into:

- Favourable risk: Gleason 3+3 (with PSA 10 to $<20 \mathrm{ng} / \mathrm{mL}$ or T2b-c) or Gleason $3+4$.

- Non-favourable risk: Gleason 3+4 (with PSA 10 to $<20 \mathrm{ng} / \mathrm{mL}$ or T2b-c) or Gleason $4+3$.

- High-risk: PSA $>20 \mathrm{ng} / \mathrm{mL}$, or Gleason 8 or greater, or clinical stage $\geq \mathrm{T} 3$.

The preferred management strategy that is widely adopted for most men with low-risk disease is AS while curative/active treatment is widely adopted for men with intermediate to high risk disease.$^{8-11}$ There is mounting evidence that men with favourable intermediate risk PC may have similar mortality risk as those with low-risk disease. These men may, therefore, be good candidates for AS, as a safe first-line management option, although the consensus to accurately define this subgroup of PC cases needs clarity. ${ }^{4-12}$

\section{Prostate cancer diagnosis}

PC is conventionally diagnosed using transrectal ultrasound to guide prostate biopsy (PB). As cancer cannot be imaged on ultrasound, the major limitation of this approach is non-detection of a substantial proportion of significant PC. Sampling error can lead to the misdiagnosis of clinically significant disease, which may be upgraded at repeat biopsy. Referred to as reclassification, this gives the perception of disease progression, subsequently leading to overtreatment. The Prostate Cancer Research International Active Surveillance Project, referred to as the PRIAS Project estimated that approximately $28 \%$ of PRIAS participants showed reclassification on repeat biopsy. ${ }^{13-15}$ The use of multiparametric MRI (mpMRI), originally used for staging, is now routinely used for tumour detection and localisation, allowing image-guided targeted sampling to overcome the limitations of the traditional blind PB. mpMRI is able to detect both high-grade and larger tumours accurately, which means it may perform particularly well for detection of clinically significant disease. ${ }^{16-18}$ It can improve the specificity of locating $\mathrm{PC}$ and targeting the $\mathrm{PB}$. It can also differentiate low- and intermediate/high-grade PC, thereby providing more accurate risk classification of PC.

\section{Prostate cancer risk and vitamin D}

Two recent clinical trials in PC patients suggest that vitamin D supplementation may prevent PC progression. ${ }^{19} 20$ Daily supplementation of $4000 \mathrm{IU}$ for 1 year reduced the number of positive cores and Gleason grade, but did not reduce PSA levels. ${ }^{19}$ A daily high dose of $40000 \mathrm{IU}$ for only 10 weeks significantly reduced PSA levels but did not change Ki67 expression in prostate tissue. Those who received $4000 \mathrm{IU}$ and $10000 \mathrm{IU}$ showed no significant reduction in PSA level. ${ }^{20}$ Duration of follow-up was short in both these trials and may have resulted in the lack of significant findings. Further trials of high vitamin $\mathrm{D}$ doses to prevent PC progression are therefore required. Trials are also needed to assess the long-term safety of vitamin D supplementation in patients with cancer.

\section{Prostate cancer and genome damage}

There is a strong link between the prevalence of markers of genome damage and cancer risk. Studies have shown that prevalence of chromosomal aberrations is 2.2-fold to 2.4-fold higher in cancer patients than in non-cancer controls, and that micronuclei (MN) formation, a marker for chromosomal instability, was associated with increased cancer incidence in a study of 6718 individuals. ${ }^{21}$ There is also evidence to link telomeres to cancer risk. ${ }^{22}$ Telomeres are repetitive TTAGGG DNA sequences that maintain genomic stability by protecting the ends of chromosomes; they shorten in length over time in normal somatic tissues due to incomplete replication of the telomere. Telomere shortening is accelerated by oxidative stress, inflammation and cell proliferation and has been linked with induction of cell senescence which guards against survival of genomically abnormal cells. ${ }^{23}$ Evidence from prospective studies show positive associations between telomere length and various cancers, including that for low-grade (OR 1.13, 95\% CI 1.01 to 1.27) and localised PC (OR 1.12, $95 \%$ CI 1.01 to 1.24 ) disease, possibly due to abnormal telomerase expression and telomere elongation, which enables the survival of genomically unstable cells, their unrestricted growth and their evolution into cancer. ${ }^{24-26}$ There is evidence to show that some micronutrients are essential to prevent genome damage but the specific impact of vitamin $\mathrm{D}$ is only just starting to be explored. ${ }^{27}$

Evidence suggests that high levels of vitamin D metabolites $\left(25(\mathrm{OH}) \mathrm{D}\right.$ and $\left.1,25(\mathrm{OH})_{2} \mathrm{D}\right)$ may prevent genome damage in leukocytes by reducing prevalence of $\mathrm{MN}$ formation and by maintaining adequate telomere length. ${ }^{28-32}$ There is also evidence to suggest that $1,25(\mathrm{OH})_{2} \mathrm{D}$ may inhibit telomerase activity in tumour tissue in a pathway involving miRNA498, a non-coding small RNA. ${ }^{33}$ This current trial provides an opportunity to concurrently examine the possible role of vitamin D in the regulation of these markers of DNA damage in PC.

\section{Rationale}

The ProsD study is a 2:1 double blinded placebo controlled randomised control trial (RCT) for high-dose vitamin D supplementation, in a group of men with PC who have 
low-to-intermediate risk of disease progression, and are undertaking AS. There is uncertainty as to whether AS or curative therapy is best for this risk group. The protocol mandates the use of MRI-detected and targeted cancers in the inclusion criteria to reduce disease reclassification bias due to sampling error on biopsy and to assist in the diagnosis of disease progression on AS.

\section{Objectives and outcomes}

The objective of this trial is to determine if monthly oral high dose vitamin D supplementation for 2 years can prevent disease progression in PC cases with lowto-intermediate risk of progression. We will assess the feasibility and safety of an initial oral loading dose of $500000 \mathrm{IU}$, followed by a monthly oral dose of $50000 \mathrm{IU}$ of cholecalciferol, for 2 years of AS.

\section{Primary outcome}

The primary trial outcome is the switch from AS to active therapy, for clinical or non-clinical reasons, within 2 years of AS.

\section{Secondary outcomes}

1. Switch from AS to active therapy, specifically for clinical reasons, within 2 years of AS.

2. Switch from AS to active therapy, specifically for nonclinical reasons, within 2 years of AS.

\section{Tertiary outcomes}

1. The proportion of PC patients achieving levels of total $25(\mathrm{OH}) \mathrm{D}$ above $75 \mathrm{nmol} / \mathrm{L}$ following vitamin $\mathrm{D}$ supplementation. We will also characterise those whose serum $25(\mathrm{OH}) \mathrm{D}$ does not increase to $75 \mathrm{nmol} / \mathrm{L}$ in response to supplementation and measure levels of $1,25(\mathrm{OH})_{2} \mathrm{D}$.

2. Extent of DNA damage in those receiving the high dose vitamin D supplementation.

3. The utility of mpMRI scan in detecting PC progression in cases managed on AS.

\section{METHODS: PARTICIPANTS, INTERVENTIONS AND OUTCOMES Participants}

Trial participants will be recruited from 15 private Australian urology clinics located in Australia, with the corresponding ethics approval being obtained for all sites. Records of all patients diagnosed in the 6 months prior will be screened by urologists and clinic nurses, to identify potential participants. Those clinically eligible will be contacted, introduced to the ProsD trial and consent obtained to forward their personal details to the trial coordinator. Each potential participant will be further screened for remaining eligibility criteria before being invited to participate. The ProsD trial will use subsets of D-Amico's classification to derive its population of interest. All eligible participants will be mailed a Participant Information Consent Form, and those who decline will not be followed up further. All consenting participants will be assigned a 7-digit identification number.

\section{Eligibility criteria}

All participants aged between 50 and $<80$ years must have results of at least one mpMRI (centrally reviewed and not limited to any PIRADS score) and one of the following:

- Gleason grade 3+4.

- >2 positive biopsy cores (which may include Gleason $6)$.

- Clinical stage T2 (which may include Gleason 6).

- PSA $>10 \mathrm{ng} / \mathrm{mL}$ (which may include Gleason 6).

Those diagnosed with these clinical features but without a prior mpMRI scan will be requested to have a scan done for the purposes of the study. Those with a pacemaker or with metal prosthesis in their body will not be asked to have an mpMRI.

Men who were previously diagnosed with low-risk disease and whose disease has been upgraded during the preceding 6 months will be eligible to participate.

\section{Exclusion criteria}

- Men with low-risk disease:

- Gleason score six or less.

- PSA $<10 \mathrm{ng} / \mathrm{mL}$.

- Clinical stage $<$ T2.

- Men with high-risk disease:

- Gleason score 8 or more.

- $\mathrm{PSA}>20 \mathrm{ng} / \mathrm{mL}$.

- Clinical stage T3 or T4 or N/M $>0$.

- Consuming daily vitamin D supplementation more than $50 \%$ of recommended dietary intake (RDI) (more than $300 \mathrm{IU} /$ day).

- Hyperparathyroidism, hypercalcaemia or osteomalacia.

- Glomerular filtration rate (GFR) below 30 or stage 4 or stage 5 kidney disease

- History of renal calculi.

- Taking orlistat, cholesterol-lowering drugs called bile acid sequestrants, such as cholestyramine and cholestipol or other drugs known to reduce vitamin D absorption.

- Those with gastrointestinal abnormalities that may affect nutrient absorption such as inability to swallow oral medication or clinically diagnosed malabsorption

\section{Sample size}

The primary outcome for the trial is the conversion from AS to active therapy within 24 months from date of consuming their initial loading dose of supplements. This is currently estimated to be $\sim 35.0 \%$ at 2 years for low-to-intermediate risk patients on AS. Using Simon's two-stage design, a sample size of 80 patients in the group receiving the vitamin $\mathrm{D}$ supplement would have $>80 \%$ power with $95 \%$ confidence to exclude a success rate of $65.0 \%$ in favour of a more interesting rate of $77.5 \%$ (ie, active therapy rate of $22.5 \%$ rather than $35.0 \%)$.

Additionally, a futility analysis will be performed after 24 patients have received vitamin D for 24 months, and 
if eight or more patients in the intervention group have disease progression after 2 years of intervention, consideration will be given to modifying the intervention or stopping the study for futility. In order to obtain contemporary estimates for the control group it is proposed to randomise patients 2:1 resulting in a total sample size of 120 patients (80 vitamin $\mathrm{D}$ supplementation, 40 placebo controls).

\section{Intervention}

The intervention is a monthly oral dose of $50000 \mathrm{IU}$ of cholecalciferol for 24 months. Participants randomised to control will take a visually identical placebo.

\section{Initial loading dose}

Each participant will be requested to orally consume 10 tablets within 12 hours commencing initial loading. They will be required to attend a pathology provider to have their blood and urine tested for toxicity the following day. They will be assessed for incidence of hypercalcaemia and hypercalcinuria, which will be measured to ensure serum and urine levels are below $2.75 \mathrm{mmol} / \mathrm{L}$ and less than $7.5 \mathrm{mmol} / 24$ hours, respectively, and to ensure the average estimated GFR is within normal range. All results will be uploaded onto the pathology providers' online results portal which will be accessed by the treating urologist and trial coordinator.

\section{Follow-up doses}

Thereafter, participants will consume one tablet a month (after 30 days), for the remaining 23 months. Each participant will be sent a reminder email and/or SMS to take the supplement a day before the due date and be followed-up the following week to ensure compliance. Each participant will also be asked questions on any lifestyle changes, their well-being, if they have had any falls, and be notified of the date of the next supplement follow-up. At the completion of intervention period, all participants will be requested to destroy all remaining supplements. ProsD procedure is outlined in figure 1 .

\section{Justification for dosing regimen}

This dosing regime is similar as that proposed by the Mel-D trial (ANZMTG 02.09 Mel-D) which is an RCT of vitamin $\mathrm{D}$ supplementation in melanoma patients. The $500000 \mathrm{IU}$ loading dose aims to achieve an early increase in $25(\mathrm{OH}) \mathrm{D}$ levels. This is followed by a monthly lower dose of $50000 \mathrm{IU}$ to maintain high levels; the monthly dosing frequency is proposed to improve the likelihood of good compliance over the trial period. This dose has been shown to achieve average serum $25(\mathrm{OH}) \mathrm{D}$ levels of $\sim 78 \mathrm{~nm} / \mathrm{L}$ without adverse effects. ${ }^{34}$

\section{Randomisation}

All supplements (placebo and active) will be packed in boxes to look similar and assigned a kit number. This information for each kit number and its corresponding content (placebo or active) will be provided to the National Health and Medical Research Council Clinical

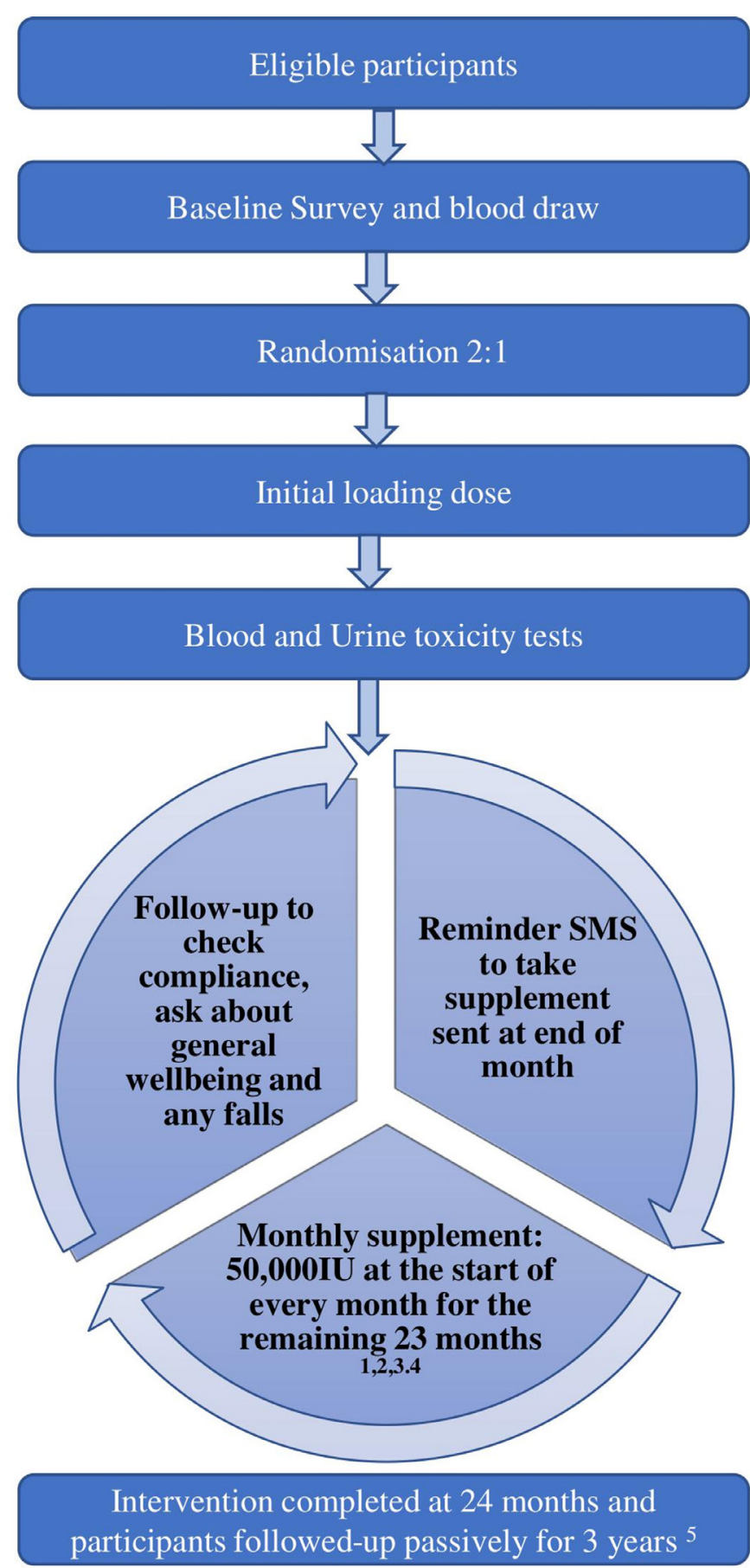

Figure 1 Overview of the ProsD Trial. PSA, prostate-specific antigen. ${ }^{1}$ At 3,6,9,12,18,24 months: Participants will have PSA and biochemical tests (at urologists' request as in standard clinical care). ${ }^{2}$ At 6,12,24 months: Participants will be reminded to have a blood draw before supplementation is due. ${ }^{3}$ At 12 and 24 months: Participants will have to complete a questionnaire and be asked to have MRI scans. ${ }^{4}$ At least one repeat biopsy during the 24 months of follow-up (at urologists' request). ${ }^{5}$ Follow-up from clinical records.

Trials Centre (NHMRC-CTC), University of Sydney, by the packaging company (PCI-Pharma). All staff at the coordinating site and treating urologists are blinded to the allocation. 
On completion of baseline data collection, participants will be randomised in the ratio of 2:1 using their 7-digit ID, date of birth, and diagnostic pathology status. Randomisation will be performed centrally at the NHMRC-CTC to guarantee allocation concealment using an interactive voice record system. Using the method of minimisation with patients stratified by age ( $\leq 65$ years, $>65$ years); Gleason score $(<7,7)$; upgraded to low-to-intermediate risk in the past 6 months (yes/no), a randomisation number will be created to generate corresponding kit numbers specific to active or placebo box of supplements. This information will be provided to the Trial Coordinator, who will then mails the assigned boxes of supplements, and a pathology request form (for blood/urine toxicity tests) to participant, with specific instructions.

\section{Endpoints}

\section{Primary endpoint}

The primary endpoint will be the proportion of participants opting for active therapy for PC (Active therapy-free survival, ATFS). There will be no absolute requirements for conversion to active therapy which will be at the discretion of the treating urologist. However, as a guideline, active therapy may be considered in the following situations.

- Gleason $4+3$ or greater on rebiopsy.

- Gleason $3+4=7$ where pattern 4 is $>10 \%$ on rebiopsy.

- Any Gleason 6 or $3+4=7$ (pattern $4<10 \%$ ) on rebiopsy where progression on mpMRI has occurred defined as (based on NCI study) ${ }^{8}$

- Increase in PIRAD score.

- Any increase in lesion diameter measured in the axial plane.

- Appearance of any new lesion.

- PSA doubling time of less than 3 years.

ATFS is defined as men who do not undergo any active therapy (eg, radical prostatectomy or radiotherapy) during the trial.

\section{Secondary endpoints}

- Proportion of participants opting for active therapy for PC for non-cancer progression (eg, anxiety).

- Proportion of participants opting for active therapy for PC for clinical reasons.

- PSA doubling time (PSA doubling time $<3$ years is progression).

- Increase in Gleason grade.

- Increase in the percentage of involved cores.

- Increase in cancer volume (total $\mathrm{mm}$ of cancer detected/total mm biopsied).

Tertiary endpoints

- Proportion of participants achieving optimal serum vitamin D levels as assessed by serum $25(\mathrm{OH}) \mathrm{D}$ levels $75 \mathrm{nmol} / \mathrm{L}$ and above.

- Prevalence of lymphocytic DNA damage as assessed by markers listed above.
- Re-evaluation of mpMRI scans in detecting PC progression in cases managed on AS.

\section{Definition of outcomes}

1. Conversion to active therapy due to clinical reasons

- Gleason $4+3$ or greater on rebiopsy.

- Gleason 3+4 $=7$ where pattern 4 is $>10 \%$ on rebiopsy.

- Any Gleason 6 or $3+4=7$ (pattern $4<10 \%$ ) on rebiopsy where progression on mpMRI has occurred defined as:

- Increase in PIRAD score.

- Any increase in lesion diameter measured in the axial plane

- Appearance of any new lesion.

- PSA doubling time of less than 3 years.

2. Conversion to active therapy due to non-clinical reasons

- Number of patients opting out of AS for non-cancer progression (eg, anxiety).

\section{Patient and public involvement}

Two consumer representatives from the Prostate Cancer Support Networks (Prostate Cancer Foundation Australia) were involved in providing feedback in the conceptual development of the study, study design, purpose and all study materials.

\section{METHODS: DATA COLLECTION AND MANAGEMENT Data collection}

Each participant will be mailed a copy of a baseline survey at the start of the project, followed by a follow-up survey at 12 months and 24 months (online supplemental appendix 1). Blood specimens will also be collected at these time points as well as at 6 months after commencement of supplementation. Each questionnaire is anticipated to take an approximately $30 \mathrm{~min}$ to complete. Participants with incomplete responses will be followed up.

For the purpose of research, blood will be collected into one heparin tube $(10 \mathrm{~mL})$ to perform the cytokinesisblock micronucleus cytome (CBMN) assay in lymphocytes, and one ethylenediaminetetraacetic acid (EDTA) tube $(10 \mathrm{~mL})$ for vitamin $\mathrm{D}$ assay. Participants will not be required to fast before blood collection. Collection will be undertaken and coordinated by Douglass Hanly Moir Pathology (DHM) in New South Wales (NSW), Sullivan and Nicolaides Pathology in Queensland and Dorevitch Pathology in Victoria. All tubes will be labelled with the 7-digit study identification number, participants' date of birth, date of blood draw and time of blood draw. Participants' names will not appear on these tubes. When blood draw is complete, specimens will be collected, packed and transported to the processing facility (Commonwealth Scientific and Industrial Research Organisation (CSIRO), Australia) within 24 hours of collection, by the courier company (Marken Australia). Date and time of blood collection and receipt of samples will be 
recorded. Blood processing protocol is outlined in online supplemental appendix 2. All participants who have not had blood drawn will be followed up with a fortnightly reminder phone call/email, for 5 weeks. Participants who do not respond after five follow-ups will be recorded as loss to follow-up.

All participants will also be mailed an MRI-request form, at 12 months and 24 months, to have this scan completed, preferably, by the same provider as that during their diagnosis.

\section{Blood processing}

All blood specimens will be processed by CSIRO laboratory (South Australia). The heparin tubes will be processed for fresh lymphocytes, and EDTA tubes will be processed to separate the plasma and buffy coats, which will be stored in $1 \mathrm{~mL}$ aliquots at $-80^{\circ} \mathrm{C}$. All tubes will be identified by their 7-digit study identification number, participants' date of birth, date of blood draw, and time of blood draw.

\section{Measurements in blood}

\section{Vitamin D assays}

All vitamin D plasma assays will be carried by DHM Laboratory, at the end of the trial. The assay for $25(\mathrm{OH}) \mathrm{D}$ will be carried out using LC-MSMS, which is specifically set up to exclude the detection of epimers. We will reassay $20 \%$ of these samples using the DiaSorin Liaison semiautomated chemiluminescence assay (DiaSorin; DEQAS accredited). Internal and external vitamin D standards will be included in each batch of analysed samples. Levels for $1,25(\mathrm{OH})_{2} \mathrm{D}$ will be measured by using the Liaison (Diasorin) platform. Mean levels for each metabolite will be measured at baseline, 6, 12 and at 24 months for each arm of the trial.

\section{Genome damage markers}

The CBMN assay will be performed using the isolated lymphocyte culture protocol described elsewhere. ${ }^{35}$ Biomarkers in the CBMN assay include formation of $\mathrm{M})$ within binucleated cells $(\mathrm{BN})$ which originate from lagging chromosome fragments or whole chromosomes during mitosis; thus the frequency of $\mathrm{BN}$ cells with $\mathrm{MN}$ (designated BN-MN) is an index of chromosome breakage and loss. Nuclear Division Index, a measure of mitogenic response, which is useful as a biomarker of immune function will also be assayed. Prevalence of DNA damage will be evaluated for each marker as per cent change from baseline to 12 months and 24 months.

\section{PSA and biopsy follow-up}

All participants will be mailed a pathology request every 3 months in the first year, and every 6 months thereafter, reminding them to have their PSA test completed. All participants are requested to have at least one repeat biopsy during the 24 months of follow-up. Information for any follow-up biopsy will be collected from their treating urologist. The timeline for blood collection for each participant is described in figure 2. This figure describes

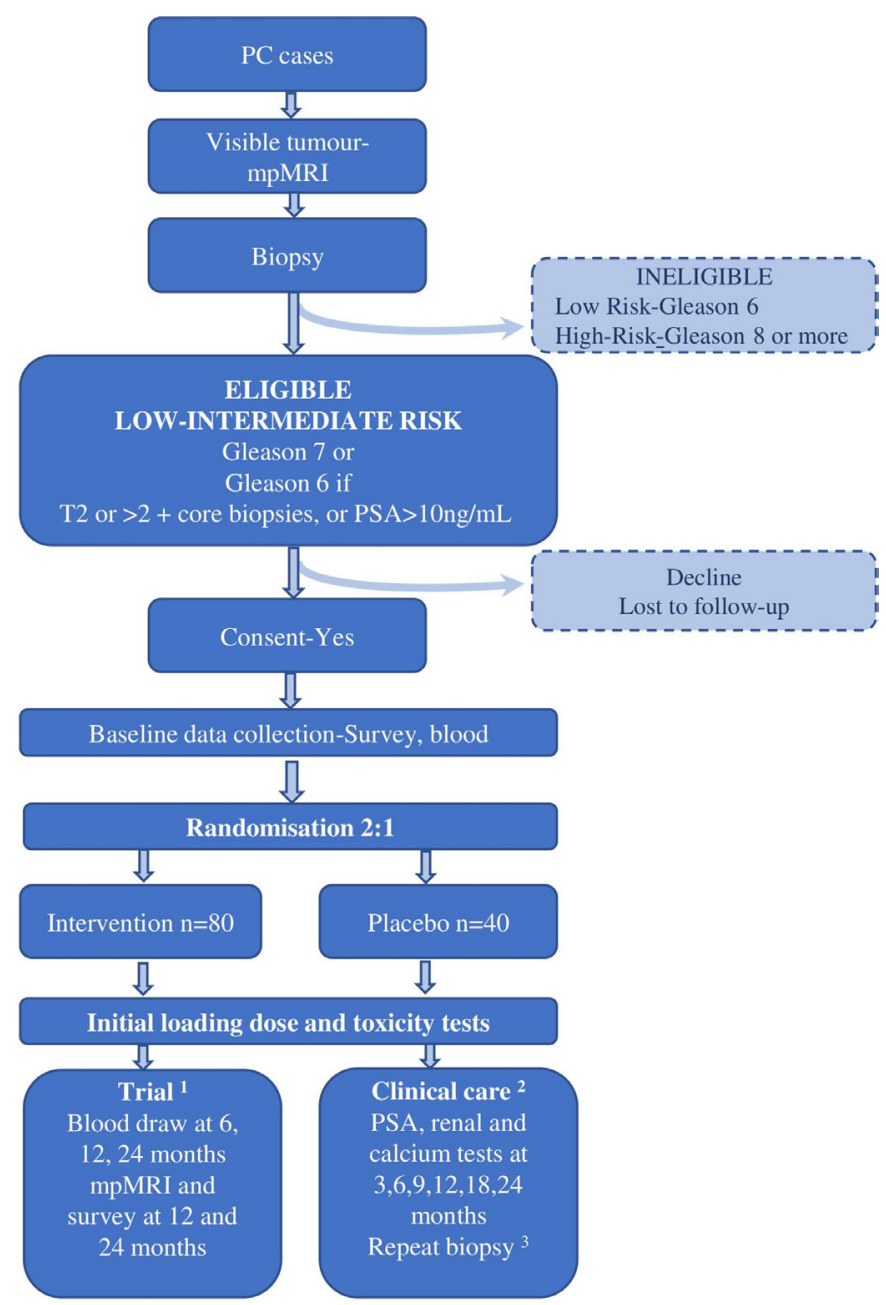

Figure 2 ProsD trial procedure. mpMRI, multiparametric MRI; PC, prostate cancer; PSA, prostate-specific antigen. ${ }^{1}$ All participants will be contacted monthly via email and SMS to take supplements, followed up one week later by phone to check compliance. ${ }^{2}$ The trial coordinator will coordinate all mail outs to ensure blood requests for clinical care coincide with that for ProsD trial requirement. ${ }^{3}$ At least one repeat biopsy during the 24 months of follow-up.

from the time point a participant is flagged as potentially eligible, through to the completion of the trial, after which all participants will continue to be followed up passively, from their clinical records obtained from their treating urologists.

Tissue biopsy analysis

Paraffin-embedded biopsy tissue will be collected and stored in licensed premises in accordance with the Human Tissue Act 1983 (NSW). We will liaise with pathologists to have slides prepared from $5 \mu \mathrm{m}$ section from biopsy tissues (diagnosis and follow-up biopsies) and assayed for Ki67 activity. Change in Ki67 proliferation from baseline biopsy to follow up biopsy will be reported.

\section{Data management}

All information collected and storage of study data will conform to the Australian Privacy Principles, Guidelines 
under Section 95A of the Privacy Act 1988. Data will be collected and stored in various formats during the trial, therefore, appropriate methods of confidentiality and privacy will be maintained. Data will be entered into a password protected database in a deidentified manner where each participant will be allocated a unique 7-digit ID number and will be archived for 10 years after the completion of the study as specified by NHMRC. All participants will be identified by their Study identification number, participants' date of birth and date of interview. All data cleaning and preparation will be conducted by research staff that are directly involved in managing the project.

A statistical analysis plan will be prepared prior to data lock and contain additional detail on the methods described below. All randomised participants will be eligible for inclusion in the full analysis set. Analysis of efficacy endpoints will be undertaken on participants in the full analysis set unless participants are deemed nonevaluable by the trial management committee; all such decisions will be documented in the final study report. While no formal comparisons between the two groups are planned, exploratory analyses will be performed to determine the level of activity of the vitamin D supplement for the key outcomes.

\section{METHODS: MONITORING AND SAFETY}

An independent data monitoring committee (IDMC) will be established to monitor the safety of participants, to ensure the continued efficacy of the trial and compliance to the trial protocol, according to the IDMC Operations Manual. There will be 12-monthly reviews conducted by the IDMC for the purpose of monitoring the conduct of the trial and assessing participant safety.

All trial participants will be monitored by the IDMC and will be immediately removed from the study if they have:

- Incidence of hypercalcaemia and hypercalcinuria, which will be measured to ensure serum and urine levels are below $2.75 \mathrm{mmol} / \mathrm{L}$ and less than $7.5 \mathrm{mmol} / 24$ hours, respectively.

- A 20\% fall in the estimated GFR, over the course of the study.

- More than two episodes of renal calculi occurring over the course of the study.

A participant who develops either hypercalcaemia or hypercalciuria will be referred to their general practitioner, for further management of their condition. A participant who develops renal calculi will be managed by their treating urologist for acute management of the stone as per normal procedures and guidelines.

\section{Participant safety}

The principal investigator will ensure that the study is completed in accordance with the guidelines set out in the National Statement on Ethical Conduct in Human Research (2007) (the National Statement) and the CPMP/ICH Note for Guidance on Good Clinical Practice.
Adverse event (AE) reporting will be recorded from the date of informed consent. In the case whereby there is an $\mathrm{AE}$, adverse reaction, serious $\mathrm{AE}$ (SAE) or suspected unexpected serious adverse reaction, an assessment of seriousness will be conducted by the PI or treating urologist. An $\mathrm{AE}$ is defined as any untoward medical occurrence (physical, psychological, social or economic), whether mild, moderate or severe, in a trial subject related to medical management, in contrast to complications of disease. An SAE is defined as any event that results in death, is life threatening, results in hospitalisation or results in disability or incapacity (persistent or significant).

Depending on the severity of the event, a medical assessment will be completed as soon as possible. It will be left to the principal investigator's clinical judgement to decide whether an $\mathrm{AE}$ is of sufficient severity to require the participant's removal from treatment. A participant may also voluntarily withdraw from treatment due to what he perceives as an intolerable AE. If either of these occurs, the participant will undergo an end of trial assessment and be given appropriate care under medical supervision until symptoms cease, or the condition becomes stable. The following will be documented and recorded in the adverse event logbook:

- Record each event as separate occurrences.

- Document participant identification number, date of birth, name.

- Document and describe the event.

- Record the start and stop times of the event.

- Document the severity of the event, mild, moderate, severe, fatal.

\section{Concomitant medications/treatments}

Concomitant medications will not be recorded during the study, except for medications used to treat adverse events or medications known to interact with the study medications. Any concomitant use of vitamin D or multivitamin supplements in sufficient detail to be able to estimate daily vitamin $\mathrm{D}$ intake from these sources (vitamin D supplementation more than $50 \%$ of RDI). Any concomitant use of calcium supplements, Orlistat or other drugs known to reduce vitamin $\mathrm{D}$ absorption will be recorded.

\section{Informed consent process}

Informed consent will be obtained prior to the participant undergoing procedures that are specifically for the purposes of the trial (online supplemental appendix 3). A copy of the signed consent form will be given to the participant to retain. The original signed form will be retained at the trial site. The right of a participant to refuse participation without giving reasons will be respected and the participant will remain free to withdraw at any time from the trial without giving reasons and without prejudicing his further treatment. The participant will be allowed as much time as wished to consider the information (within time frame of eligibility criteria), and the opportunity to question the investigator or other independent parties to decide whether they will participate in the trial. Potential 
participants will only be approached for the study if they have the full capacity to give consent and that they understand the implications of the trial. There will be no provision for alternatives to obtaining consent via the Guardianship Division within NSW Civil and Administrative Tribunal. Non-respondents will be followed up five times, fortnightly and will not be followed up thereafter.

\section{Ethics and dissemination}

The protocol for the ProsD trial and all its associated documents has been reviewed and approved by the Bellberry Human Research Ethics Committee (2016-06-459-A1), and the Macquarie University Human Research Ethics Committee (5201700188). The trial will be conducted in compliance with the approved trial protocol. Any deviations from the protocol will be first submitted to Bellberry HREC for review and approval. Protocol violations will be immediately reported to the ethics committee according to its standard policies and procedures.

Any deviations from the protocol will be first submitted to Bellberry HREC for review and approval. Protocol violations will be immediately reported to the ethics committee according to its standard policies and procedures

\section{Study progress}

Recruitment was temporarily stopped in March 2020 due to the COVID-19 outbreak and was resumed in July 2020. Rate of recruitment was slow in the initial stages of the trial and hence modifications were made to the protocol to improve this rate. The original requirement to include PC cases diagnosed in the preceding 3 months was revised to include cases diagnosed in the preceding 6 months, to extend the maximum age cut-off from 75 years to 79 years, and to include PC cases who were originally diagnosed with low-risk disease but were later upgraded to low-to-intermediate risk in a follow-up biopsy. Additionally, the original requirement to have biopsies at 12 months and 24 months was changed to have at least one biopsy in the 24 months of follow-up. As of August 2020, the ProsD trial had recruited 123 participants, bypassing the prespecified target number.

\section{Author affiliations}

${ }^{1}$ Cancer Research Division, Cancer Council New South Wales, Woolloomooloo, New South Wales, Australia

${ }^{2}$ Faculty of Medicine and Health Sciences, Macquarie University, Sydney, New South Wales, Australia

${ }^{3}$ Faculty of Medicine and Health, University of Sydney, Sydney, New South Wales, Australia

${ }^{4}$ Clinical Trials Center, The University of Sydney, Sydney, New South Wales, Australia ${ }^{5}$ Discipline of Surgery, The University of Sydney, Sydney, New South Wales, Australia

${ }^{6}$ Urology, Monash Health, Clayton, Victoria, Australia

${ }^{7}$ Centre for Clinical Research, The University of Queensland, Saint Lucia, Queensland, Australia

${ }^{8}$ Wesley Urology Clinic, Wesley Hospital, Brisbane, Queensland, Australia

${ }^{9}$ Faculty of Health, Queensland University of Technology, Brisbane, Queensland, Australia

${ }^{10} \mathrm{Clinical}$ and Health Sciences, University of South Australia, Adelaide, South Australia, Australia

${ }^{11}$ Sydney Adventist Hospital Clinical School, The University of Sydney, Sydney, New South Wales, Australia
${ }^{12}$ School of Public Health, The University of Western Australia, Perth, Western Australia, Australia

${ }^{13}$ Urology, North Shore Private Hospital, St Leonards, New South Wales, Australia

${ }^{14}$ Urology Centre, Port Macquarie, New South Wales, Australia

${ }^{15}$ Rural Clinical School, University of New South Wales, Sydney, New South Wales, Australia

Acknowledgements The authors would like to acknowledge the following members for their contribution to the ProsD trial: Patient/Consumer representatives Mr Goodall and Mr Casey for their feedback in the development of this trial, to all participating urologists for enabling recruitment, the secretariat at the NHMRC Clinical Trial Centre Ms Cochrane, trial coordination by Ms Rodger and Ms Foo, and for the research assistance by Ms Willis and Ms Ozersky. The authors thank all the men participating in the ProsD trial.

Contributors $\mathrm{HG}$ is the principal investigator of ProsD. VN-S, MiF and BKA conceived the initial concept of this trial and in conjunction with HG, DPS, MIP, JWY, $\mathrm{MaF}, \mathrm{DG}, \mathrm{RG}$ and MGK developed the rationale for this trial. VN-S and HG led the development of the trial protocol and drafted this manuscript. VN-S and DPS were involved in coordinating all aspects of this trial, data collection and management. VG and DE were responsible for the statistical design of the trial, protocol development and setup the IVRS system for blinding and randomising participants. MiF was responsible for the processing of all blood specimens and undertaking the genome damage assays. MIP, MaF, JWY, DG, HW, KR, NA and JS contributed to recruiting high volume of participants to this trial. All authors have contributed to the development of the study protocol and this manuscript.

Funding This work was funded by the Movember Clinical Trials Award and administered through the Prostate Cancer Foundation Australia (CTA1315).

\section{Competing interests None declared.}

Patient and public involvement statement Two consumer representatives from the Prostate Cancer Support Networks (Prostate Cancer Foundation Australia), were involved in providing feedback in the conceptual development of the study, study design, purpose, and all study materials.

\section{Patient consent for publication Not required.}

Ethics approval This trial is approved by Bellberry Ethics Committee (2016-06459). All results will be reported in peer-reviewed journals.

Provenance and peer review Not commissioned; externally peer reviewed.

Supplemental material This content has been supplied by the author(s). It has not been vetted by BMJ Publishing Group Limited (BMJ) and may not have been peer-reviewed. Any opinions or recommendations discussed are solely those of the author(s) and are not endorsed by BMJ. BMJ disclaims all liability and responsibility arising from any reliance placed on the content. Where the content includes any translated material, BMJ does not warrant the accuracy and reliability of the translations (including but not limited to local regulations, clinical guidelines, terminology, drug names and drug dosages), and is not responsible for any error and/or omissions arising from translation and adaptation or otherwise.

Open access This is an open access article distributed in accordance with the Creative Commons Attribution Non Commercial (CC BY-NC 4.0) license, which permits others to distribute, remix, adapt, build upon this work non-commercially, and license their derivative works on different terms, provided the original work is properly cited, appropriate credit is given, any changes made indicated, and the use is non-commercial. See: http://creativecommons.org/licenses/by-nc/4.0/.

Author note Coordinating centre: Dr Nair-Shalliker and A/Prof Smith at Cancer Council NSW coordinated all aspects of this trial. They contributed to study design and protocol development, data collection, management, analysis, interpretation of results, writing of the report, and the decision to submit the report for publication. Steering committee: Professor Gurney, Dr Nair-Shalliker, A/Prof Smith, Professor Patel, Professor Woo, Professor Gebski, Mr Espinoza, Professor Yaxley, Professor Gardiner, Professor Frydenberg and Professor Gillatt, all contributed to the study design, decision making, interpretation of data, writing of the report, and the decision to submit the report for publication. Independent Data Monitoring and Safety Committee: Dr Hayden, A/Prof Wilcken and Dr Asher. Radiology assessment panel: Associate Professor Richard O'Sullivan, Dr Alain Lavoipierre and Dr Lisa Tarlinton assessed the validity of the routine radiology reports on the MRI scans. Data Management team: Dr Nair-Shalliker, A/Prof Smith, Ms Rodger and Ms Foo. Blinding and Randomisation of participants: Professor Gebski and Mr Espinoza from the NHMRC Clinical Trials Centre setup the Interactive Voice Response System (IVRS) for blind randomisation of participants. Blood collection, transportation and 
processing: All participants were requested to attend their nearest Sonic Healthcare pathology for blood sampling. Blood specimens collected to monitor PSA levels were sent to the Sonic central processing laboratory for analysis. Blood specimens collected for the purpose of the trial, were transported by Marken Australia to the processing laboratory. The Commonwealth Scientific and Industrial Research Organisation (CSIR0-Adelaide) was responsible for processing and storing all blood specimens for analysis at end of trial. Pharmaceutical manufacture and packaging: Vitamin $D$ supplements and placebo were initially manufactured and packaged by API Consumer Brands (NZ). A second batch was manufactured by BioTech Pharmacal Inc (USA) and packaged by PCI Pharma (Australia).

ORCID iD

Visalini Nair-Shalliker http://orcid.org/0000-0001-6277-5125

\section{REFERENCES}

1 Bray F, Ferlay J, Soerjomataram I, et al. Global cancer statistics 2018: GLOBOCAN estimates of incidence and mortality worldwide for 36 cancers in 185 countries. CA Cancer J Clin 2018;68:394-424.

2 Ferlay J, Colombet M, Soerjomataram I, et al. Estimating the global cancer incidence and mortality in 2018: GLOBOCAN sources and methods. Int J Cancer 2019;144:1941-53.

3 Gulati R, Cheng HH, Lange $\mathrm{PH}$, et al. Screening men at increased risk for prostate cancer diagnosis: model estimates of benefits and harms. Cancer Epidemiol Biomarkers Prev 2017;26:222-7.

4 Hamdy FC, Donovan JL, Lane JA, et al. 10-Year outcomes after monitoring, surgery, or radiotherapy for localized prostate cancer. $N$ Engl J Med 2016;375:1415-24.

5 Clinically localized prostate cancer: AUA/ASTRO/SUO guideline, 2017. Available: https://www.auanet.org/guidelines/prostate-cancerclinically-localized-guideline

6 Sanda MG, Cadeddu JA, Kirkby E, et al. Clinically localized prostate cancer: AUAVASTRO/SUO guideline. Part I: risk stratification, shared decision making, and care options. J Urol 2018;199:683-90.

7 Thompson I, Thrasher JB, Aus G, et al. Guideline for the management of clinically localized prostate cancer: 2007 update. $J$ Urol 2007;177:2106-31.

8 Bekelman JE, Rumble RB, Chen RC, et al. Clinically localized prostate cancer: ASCO clinical practice guideline endorsement of an American urological Association/American Society for radiation Oncology/Society of urologic oncology guideline. J Clin Oncol 2018;36:3251-8.

9 Mohler JL, Antonarakis ES, Armstrong AJ, et al. Prostate cancer, version 2.2019, NCCN clinical practice guidelines in oncology. J Natl Compr Canc Netw 2019;17:479-505.

10 Mottet N, Bellmunt J, Bolla M, et al. EAU-ESTRO-SIOG guidelines on prostate cancer. Part 1: screening, diagnosis, and local treatment with curative intent. Eur Urol 2017;71:618-29.

11 Sanda MG, Cadeddu JA, Kirkby E, et al. Clinically localized prostate cancer: AUA/ASTRO/SUO guideline. Part II: recommended approaches and details of specific care options. J Urol 2018;199:990-7.

12 Dall'Era MA, Klotz L. Active surveillance for intermediate-risk prostate cancer. Prostate Cancer Prostatic Dis 2017;20:1-6.

13 Bul M, Zhu X, Valdagni R, et al. Active surveillance for lowrisk prostate cancer worldwide: the PRIAS study. Eur Urol 2013;63:597-603.

14 Loeb S, Walter D, Curnyn C, et al. How active is active surveillance? intensity of followup during active surveillance for prostate cancer in the United States. J Urol 2016;196:721-6.

15 Kalapara AA, Verbeek JFM, Nieboer D, et al. Adherence to active surveillance protocols for low-risk prostate cancer: results of the
Movember Foundation's global action plan prostate cancer active surveillance initiative. Eur Urol Oncol 2020;3:80-91.

16 Fütterer JJ, Briganti A, De Visschere P, et al. Can clinically significant prostate cancer be detected with multiparametric magnetic resonance imaging? A systematic review of the literature. Eur Urol 2015;68:1045-53.

17 Panebianco V, Barchetti F, Sciarra A, et al. Multiparametric magnetic resonance imaging vs. standard care in men being evaluated for prostate cancer: a randomized study. Urol Oncol 2015;33:17.e1-7.

18 Donato P, Morton A, Yaxley J, et al. Improved detection and reduced biopsies: the effect of a multiparametric magnetic resonance imaging-based triage prostate cancer pathway in a public teaching hospital. World J Urol 2020;38:371-9.

19 Marshall DT, Savage SJ, Garrett-Mayer E, et al. Vitamin D3 supplementation at 4000 international units per day for one year results in a decrease of positive cores at repeat biopsy in subjects with low-risk prostate cancer under active surveillance. J Clin Endocrinol Metab 2012;97:2315-24.

20 Wagner D, Trudel D, Van der Kwast T, et al. Randomized clinical trial of vitamin D3 doses on prostatic vitamin D metabolite levels and Ki67 labeling in prostate cancer patients. J Clin Endocrinol Metab 2013;98:1498-507.

21 Bonassi S, Hagmar L, Strömberg U, et al. Chromosomal aberrations in lymphocytes predict human cancer independently of exposure to carcinogens. European Study Group on cytogenetic biomarkers and health. Cancer Res 2000;60:1619-25.

22 Liu JJ, Prescott J, Giovannucci E, et al. Plasma vitamin D biomarkers and leukocyte telomere length. Am J Epidemiol 2013;177:1411-7.

23 Campisi J, Robert L. Cell senescence: role in aging and age-related diseases. Interdiscip Top Gerontol 2014;39:45-61.

24 Julin B, Shui I, Heaphy CM, et al. Circulating leukocyte telomere length and risk of overall and aggressive prostate cancer. $\mathrm{Br} J$ Cancer 2015;112:769-76.

25 Julin B, Shui IM, Prescott J, et al. Plasma vitamin D biomarkers and leukocyte telomere length in men. Eur J Nutr 2017;56:501-8.

26 Yuan X, Larsson C, Xu D. Mechanisms underlying the activation of TERT transcription and telomerase activity in human cancer: old actors and new players. Oncogene 2019;38:6172-83.

27 Fenech MF. Dietary reference values of individual micronutrients and nutriomes for genome damage prevention: current status and a road map to the future. Am J Clin Nutr 2010;91:1438S-54.

28 Nair-Shalliker V, Armstrong BK, Fenech M. Does vitamin D protect against DNA damage? Mutat Res 2012;733:50-7.

29 Nair-Shalliker V, Dhillon V, Clements M, et al. The association between personal sun exposure, serum vitamin D and global methylation in human lymphocytes in a population of healthy adults in South Australia. Mutat Res 2014;765:6-10.

30 Nair-Shalliker V, Fenech M, Forder PM, et al. Sunlight and vitamin $\mathrm{D}$ affect DNA damage, cell division and cell death in human lymphocytes: a cross-sectional study in South Australia. Mutagenesis 2012;27:609-14.

31 Beilfuss J, Camargo CA, Kamycheva E. Serum 25-hydroxyvitamin D has a modest positive association with leukocyte telomere length in middle-aged us adults. J Nutr 2017;147:514-20.

32 Mazidi M, Michos ED, Banach M. The association of telomere length and serum 25-hydroxyvitamin D levels in US adults: the National health and nutrition examination survey. Arch Med Sci 2017;13:61-5.

33 Kasiappan R, Shen Z, Tse AK-W, et al. 1,25-Dihydroxyvitamin D3 suppresses telomerase expression and human cancer growth through microRNA-498. J Biol Chem 2012;287:41297-309.

34 Tran B, Armstrong BK, Carlin JB, et al. Recruitment and results of a pilot trial of vitamin $D$ supplementation in the general population of Australia. J Clin Endocrinol Metab 2012;97:4473-80.

35 Fenech M. Cytokinesis-block micronucleus cytome assay. Nat Protoc 2007;2:1084-104. 